\title{
Zukünftigen Herausforderungen wirkungsvoll begegnen
}

\section{Bruno Kesseli, Text und Bilder}

Dr. med. et lic. phil., Chefredaktor

Mit den «Perspektiven der ärztlichen Bildung» befasste sich das zweite MedEd Symposium des SIWF am 23. September 2015 in Bern. Die Veranstaltung wurde dem Anspruch gerecht, Experten zu einem intensiven Austausch über aktuelle Fragen der ärztlichen Bildung zusammenzubringen und (Denk-)Anstösse zu vermitteln, um in diesem Bereich für die absehbaren zukünftigen Herausforderungen an das medizinische Versorgungssystem gewappnet zu sein.

"Wir wollen nicht nur eine Verwaltungsstelle sein", hielt SIWF-Präsident Werner Bauer zum Auftakt des 2. MedEd Symposiums fast schon programmatisch fest. Zwar gehören administrative Aufgaben ohne Zweifel zum Pflichtenheft des Schweizerischen Instituts für ärztliche Weiter- und Fortbildung, doch dem Präsidenten ist es ein explizites Anliegen, das SIWF auch als aktiven Mitgestalter der ärztlichen Bildungslandschaft zu positionieren. Mit dem 2. MedEd Symposium, so viel sei vorweggenommen, ist ihm dies gelungen. Die Veranstaltung bot einige bemerkenswerte Auftritte, in denen Ideen präsentiert wurden, die einer vertieften Diskussion und Weiterentwicklung würdig scheinen.

\section{Vielfältige Aktivitäten des SIWF}

Neben dem allgemeinen Informations- und Gedankenaustausch nannte Werner Bauer als Ziele des Symposiums das «Erkennen und Analysieren von Problemen und Herausforderungen", die «Diskussion verschiedener Optionen für die weitere Gestaltung von Weiterund Fortbildung», das «Besprechen methodischer Neuerungen» und schliesslich den «Blick ins Ausland».

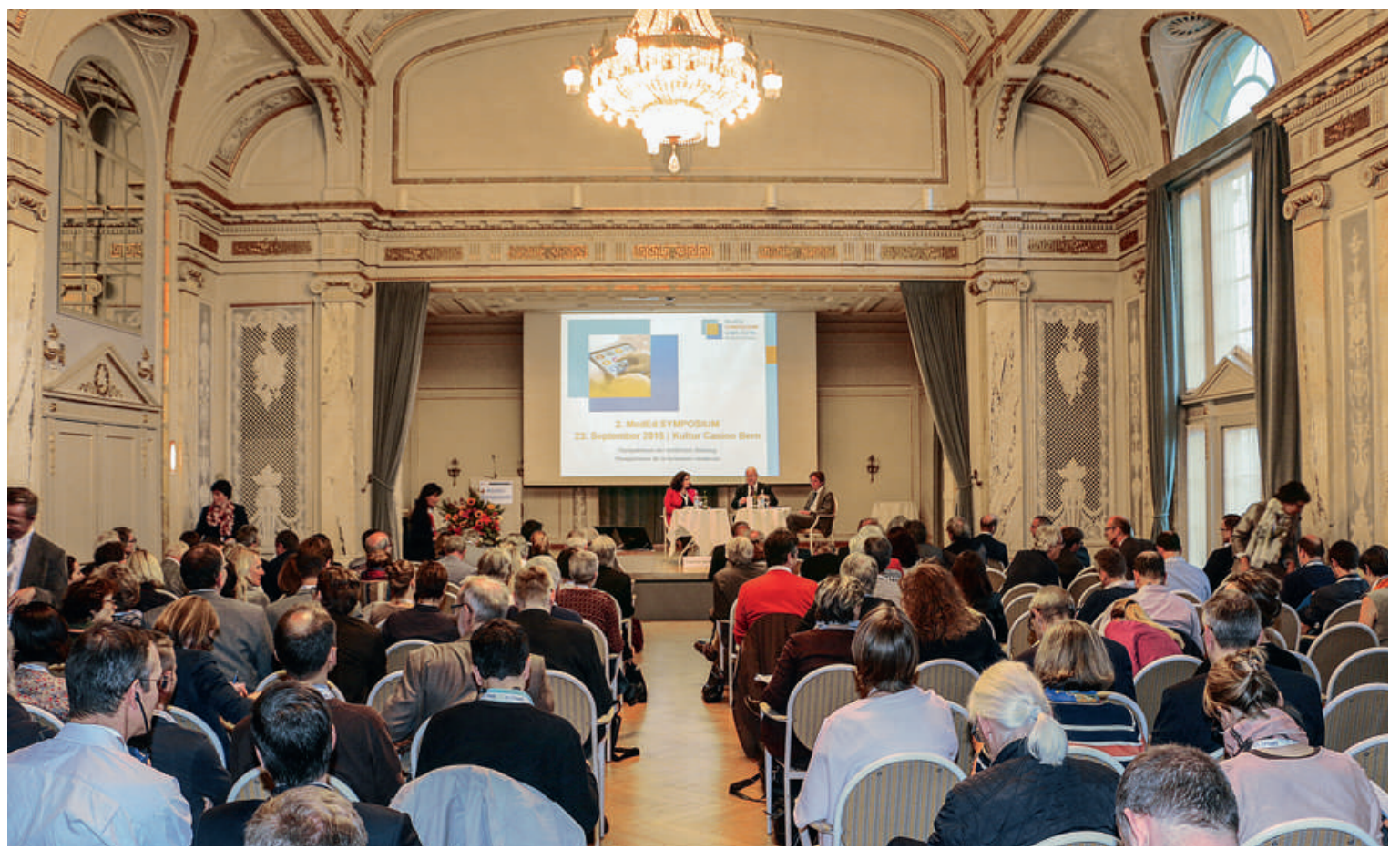

Auch in diesem Jahr erwies sich das MedEd Symposium als zugkräftiger Anlass für ein fachkundiges Publikum. 
Er wartete mit einer Reihe beeindruckender Zahlen auf, die die vielfältigen Aktivitäten des SIWF belegen. Das Institut ist für 10000 Assistenzärztinnen und -ärzte und 1500 Weiterbildungsstätten zuständig, hat 100 Weiterbildungsprogramme auszuarbeiten, zu aktualisieren und umzusetzen, jährlich 2000 Titelgesuche und 1000 Anfragen ausländischer Ärztinnen und Ärzte zu bearbeiten und 100 Visitationen durchzuführen, um nur einige seiner Aufgaben numerisch zu charakterisieren. Die Anzahl der erteilten Facharzttitel nahm in den letzten Jahren kontinuierlich zu und lag im vergangenen Jahr bei 1501. 44 Prozent der Titel wurden an Ärztinnen und Ärzte mit einem ausländischen Arztdiplom vergeben. Mit 62 Prozent stammt der Löwenanteil davon aus Deutschland, gefolgt von Österreich (12\%) und Italien (7\%). Diese Zahlen zeigen, dass die (überwiegende) Deckung des Bedarfs durch hierzulande ausgebildete Ärztinnen und Ärzte noch in weiter Ferne ist. Als weitere Herausforderungen identifizierte Werner Bauer beispielsweise Veränderungen von Strukturen im Gesundheitswesen und in der Gesellschaft, den Stellenwert des "Teachings», die Finanzierung der Weiterbildung, das Spannungsfeld von Lernen versus Dienstleistung, neue Wege der Lernzieldefinition, sinnvolle Evaluationsmethoden oder die Steuerung von Weiterbildung und Niederlassung.

\section{Koordination statt Steuerung}

Diesen letzten Punkt, der einigen gesundheitspolitischen Zündstoff in sich birgt, griff auch Pascal Strupler in seinem Referat auf. Die Lösung für das Problem, dass die Verteilung der Ärzteschaft hierzulande teilweise nicht bedarfsgerecht ist, sieht der Direktor des Bundesamts für Gesundheit BAG allerdings nicht in

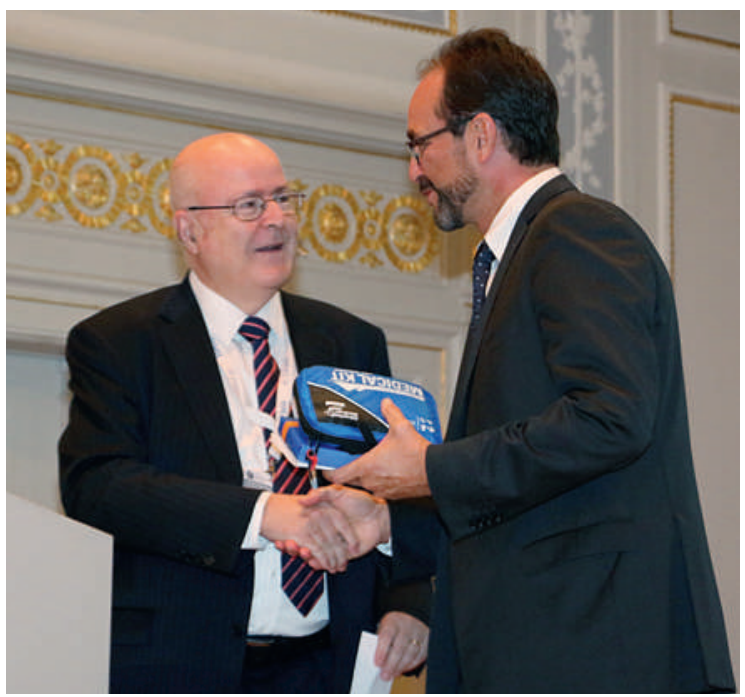

Kleine Geschenke erhalten die Freundschaft: Werner Bauer (links) dankt Pascal Strupler für dessen Referat.

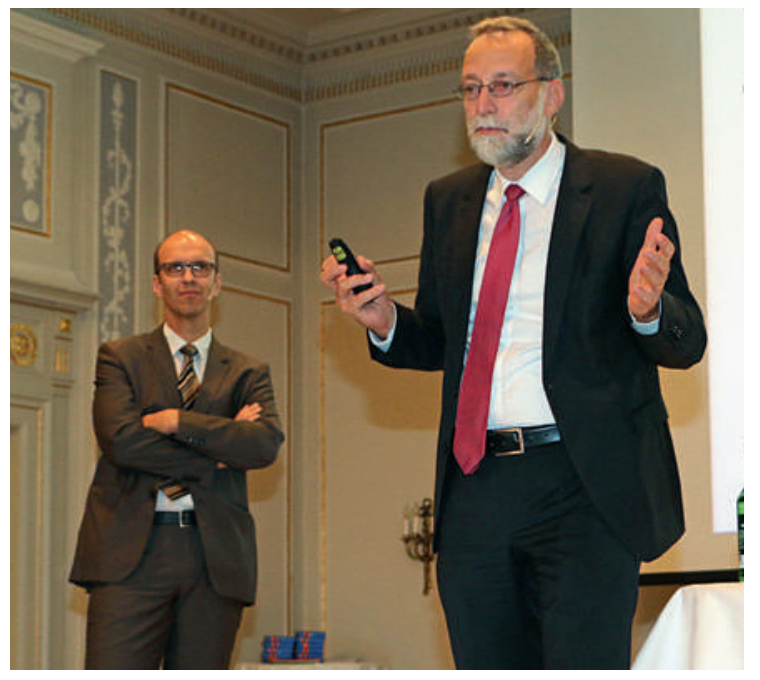

Gutes Zusammenspiel: Rolf Zehnder (rechts) und Stefan Breitenstein sprachen zur ärztlichen Weiterbildung im Spitalumfeld.

einer zentralistischen Steuerung über Quoten, die er in der Schweiz ohnehin nicht für realisierbar hält. «Wir müssen andere Wege finden", meinte er und ortete beispielsweise Verbesserungspotential bei der Koordination der Gesundheitsversorgung und im Bereich der interprofessionellen Zusammenarbeit. Er wies auch auf die unbefriedigende Datenlage im Bereich der ambulanten Versorgung und die Anstrengungen des BAG hin, «Licht ins Dunkel zu bringen». Diese Daten seien wesentlich im Hinblick auf die Bewältigung der anstehenden Herausforderungen wie steigender Lebenserwartung und damit zusammenhängender Zunahme chronischer Krankheiten, unterstrich er, ortete aber in der Ärzteschaft auch Ängste vor zu viel Transparenz. Was den Mangel an «eigenem» ärztlichen Nachwuchs betrifft, ist Pascal Strupler optimistisch. Er verwies neben der bereits realisierten Erhöhung der Anzahl Ausbildungsplätze auf künftige Angebote der ETH Zürich, in Fribourg, St. Gallen und im Tessin. Irgendwann, so seine Prognose, werde nicht mehr über zu wenige, sondern über zu viele Ausbildungsplätze diskutiert werden. Das SIWF sieht der BAG-Direktor als zentrales Bindeglied zwischen den verschiedenen in die ärztliche Bildung involvierten Akteuren. Er war sich mit Werner Bauer darin einig, dass die Zusammenarbeit zwischen BAG und SIWF gut sei. Ganz nach dem Motto «Was sich liebt, das neckt sich» konterte er denn auch Werner Bauers Randbemerkung, im BAG seien Ärzte mittlerweile Mangelware. Wenn dem so wäre, meinte er entspannt, hätte es vielleicht etwas mit der Attraktivität des TARMED zu tun ...

\section{Dynamisches Winterthurer Duett}

Anschauungsunterricht dafür, wie ein gutes Zusammenspiel zwischen «Verwaltung» und «Praxis» aus- 
Die Präsentationen zu den Hauptreferaten und Seminarien sind unter www.fmh.ch/bildung-siwf/ themen/meded-sympo sium.html zugänglich. sehen könnte, bot der Auftritt von Rolf Zehnder und Stefan Breitenstein. "Ärztliche Weiterbildung: Motor oder Bremsklotz für die Entwicklung der Spitäler»* lautete das Thema, das Zehnder in seiner Funktion als Direktor des Kantonsspitals Winterthur (KSW) vorgegeben worden war. Um den Bezug zur praktischen ärztlichen Tätigkeit zu gewährleisten, holte er mit Stefan Breitenstein kurzfristig den Departementsdirektor Chirurgie des KSW mit an Bord, was den Ausführungen zu zusätzlicher Dynamik verhalf. Ihr Referat war ein überzeugtes - und überzeugendes - Plädoyer dafür, der ärztlichen Weiterbildung im Spitalumfeld unter sich rasch verändernden Rahmenbedingungen (weiterhin) einen hohen Stellenwert zuzumessen.

Auch unter strategischen Gesichtspunkten, so eine Kernaussage, sei die ärztliche Weiterbildung für die Spitäler und die Spitalbranche im Hinblick auf deren Positionierung essentiell, wobei der Vernetzung mit Bildungsinstitutionen eine wachsende Bedeutung zukomme. Ihre Vorstellungen «zur fruchtbaren Entwicklung der ärztlichen Weiterbildung an Spitälern» fassten sie in sieben Thesen ebenso prägnant wie anschaulich zusammen. "Wir wollen weiterbilden müssen», hiess es etwa unter "These 1: Ärztliche Weiterbildung ist Aufgabe der Spitäler». Die Branche hätte sich zu lange um die ärztliche Weiterbildung foutiert, wurde (selbst)kritisch angemerkt, aber da sie letztlich für den eigenen Bedarf ausbilde, müsse sie sich auch für diese Aufgabe zuständig fühlen. Die heutige strukturbezogene Weiterbildung sei zugunsten fähigkeitsbezogener Modelle aufzugeben und neue Berufsbilder wie dasjenige der Clinical Nurse oder des Physician Assistant seien zu fördern, da sie letztlich zu attraktiveren Curricula für Ärzte in Weiterbildung führten. Die besten Rahmenbedingungen für eine optimale Weiterbildung bieten aus ihrer Sicht Netzwerke und Konzernstrukturen. Auch in ihrem Modell hätte das SIWF eine zentrale Rolle, sofern es als starke und unabhängige Institution in einer institutionalisierten Zusammenarbeit mit den Spitälern und den weiteren Akteuren im ärztlichen Bildungsbereich agieren könnte.

\section{SIWF-Award für besonderes Engagement in der Weiterbildung} Im Rahmen des 2. MedEd Symposiums präsentierte Werner Bauer auch kurz die Gewinner 2015 des SIWF-Awards für besonderes Engagement in der Weiterbildung, der aufgrund der Nomination durch ehemalige Assistenzärztinnen und -ärzte vergeben wird. Folgende Weiterbildner erhielten in diesem Jahr eine Auszeichnung:

Beat Althaus, Zürich; Christian Begemann, Schaffhausen; Marco Conti, Lugano; Andreas Günthert, Luzern; Martina Hafner, Basel; Evelyn Hartmann-Mohr, Thun; Lukas Hefermehl, Baden; Christian Kellenberger, Zürich; Christoph Kniestedt, Zürich; Andreas Kühne, Buchs; François Kundig, La Chaux-de-Fonds; Sébastien Martin, Lausanne; Thorsten Meuthen, Altstätten; Thi Dan-Linh Nguyen-Kim, Zürich; Sebastian Olbrich, Zürich; Olivier Pasche, Thierrens; François Scerba, Nyon; Reto Sutter, Zürich; Sebastian Walther, Bern; Susanne Wegener, Zürich; Rainer Wolf, Bern; Wolfgang Zaunbauer, St. Gallen.

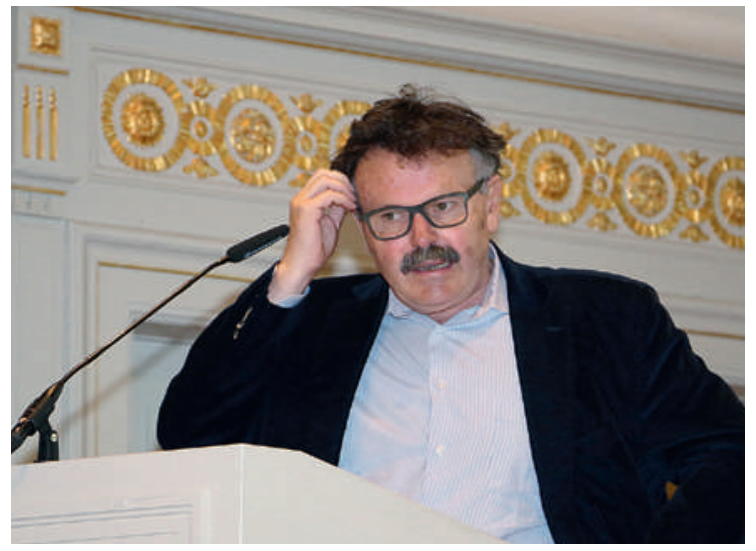

Neubau unumgänglich: Johann Steurer möchte die Ausbildung von überflüssigem Ballast befreien.

\section{Fachidiotie als Charaktereigenschaft}

«Die Weiterbildung sanft renovieren oder auf den Kopf stellen?» lautete die Frage, die Johann Steurer in seinem Referat zu beantworten hatte. Der Direktor des HortenZentrums für praxisorientierte Forschung und Wissenstransfer der Universität Zürich tendierte eindeutig zu Letzterem. Das Prinzip sanfte Renovation habe nicht funktioniert, stellte er fest, so dass ein Neubau unumgänglich sei. Das immer noch verbreitete Daten- und Faktenwissen bringe nichts, und es sei eine Wahnvorstellung, zu meinen, man könne Ärzte ausbilden, die die gesamte Medizin überblicken. Heute seien Ärztinnen und Ärzte bei der Erlangung ihres Facharzttitels durchschnittlich 37-jährig. Dieses Ziel müsste seiner Meinung nach spätestens im Alter von 30 Jahren erreicht sein. Sein Lösungsvorschlag setzt bei der Ausbildung an, die er von überflüssigem Ballast befreien möchte: Im Grundstudium (Bachelorstufe) solle das vermittelt werden, was alle brauchten - "was auch immer das dann ist» - während auf Masterstufe die Spezialisierung eingeleitet würde. Die wichtigsten Gegenargumente nahm er gleich selbst vorweg, um sie dann zu entkräften. Der Befürchtung, die Spezialisierung erfolge in diesem Modell zu früh, hielt er entgegen, angehende Lehrlinge müssten solche Entscheidungen bereits im Alter von 15 Jahren treffen. Auch die Gefahr, auf diese Weise das Fachidiotentum zu fördern, sieht er nicht: «Fachidiotie ist eine Charaktereigenschaft», meinte er. Bei entsprechend disponierten Individuen sei dagegen kein (Ausbildungs-)Kraut gewachsen. Wie die Pausendiskussionen zeigten, sorgten Steurers Überlegungen durchaus für Gesprächsstoff - es wird interessant sein, allfällige Langzeitauswirkungen zu beobachten.

\section{Lobende Worte für die Romandie}

Ganz harmonisch ging es in der Folge beim MedEdInterview zu und her, das Werner Bauer zum Thema 


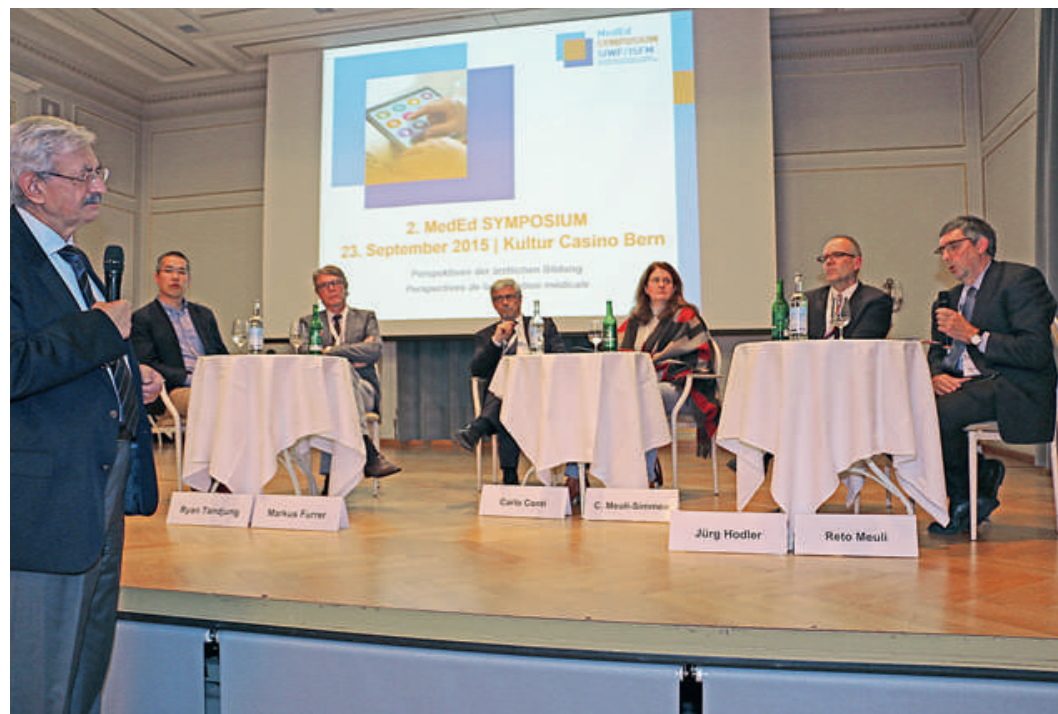

Pointierte Stellungnahmen: Iwan Rickenbacher (links) und seine Podiumsgäste.

"Ärztliche Bildung - die Rolle von Bund und Kantonen" mit Catherine Gasser und Michael Jordi führte. Sowohl die Abteilungsleiterin Gesundheitsberufe des BAG als auch der Zentralsekretär der Schweizerischen Gesundheitsdirektorenkonferenz (GDK) bekannten sich zu einem «koordinierten Föderalismus», in dem der Dialog zwischen den Akteuren von entscheidender Bedeutung sei. Letztlich seien alle Partner auf Gedeih und Verderb aufeinander angewiesen, hielt Jordi fest, und schloss damit auch das SIWF und die FMH ein. Wie schon Pascal Strupler lobte Catherine Gasser die gute Zusammenarbeit mit dem SIWF, während sie bei der FMH grosse Spannungen zwischen Generalisten und Spezialisten wahrnimmt. Daraus würden gelegentlich Abwehrreflexe wie die Gleichsetzung "Berset = Staatsmedizin» resultieren. Durch solche Vereinfachungen würden Chancen im Hinblick auf Innovationen und gute, gemeinsam erarbeitete Lösungen vertan. Lobende Worte fand Gasser für die Romandie, der sie hinsichtlich Kooperation und Vorwärtsstrategien im Bereich von Aus-, Weiter- und Fortbildung innerhalb der Schweiz eine Vorreiterrolle attestierte.

\section{Möge der Weg holprig bleiben}

In gewohnt geistreich-witziger Art setzte sich der Philosoph und Publizist Ludwig Hasler mit dem «holprigen Weg vom Lernziel zur Kompetenz» (so der leicht umgestellte Titel seines Referats) auseinander. Er gab seiner Hoffnung Ausdruck, der Weg möge holprig bleiben, und stellte die Frage in den Raum, was denn eigentlich "der richtige Mensch" sei. Als ein wesentliches Merkmal identifizierte er die fehlende Eindeutigkeit des Menschen, der stets "Justierungs- sollten nicht einfach Ministranten des Zeitgeistes sein sondern ihre Vorstellungen von Heilkunst verwirklichen können. Es gäbe elementare Fähigkeiten des Arztes, die nicht in Lernzielen vermittelt werden könnten. "Ich rede von etwas, das ich sein muss, das ich nicht einfach haben kann.» Was Aus-, Weiter- und Fortbildung betrifft, plädierte Hasler für «mehr Denken» und «mehr Konfrontation», wobei er als Beispiel die ETH nannte, die viel für die Partnerschaft von heterogenen Denkweisen mache. Lernen im Sinne von «Informationen in sich ablagern" sollte man vergessen, empfahl er.

\section{Flexiblere Weiterbildungskonzepte durch ökonomischen Druck?}

Der Nachmittagsteil wurde durch vier parallel geführte Seminarien* eingeleitet, die folgende Themen behandelten:

- den Strukturwandel im Gesundheitswesen und die Weiterbildung;

- Lernzielformulierung, Assessment, Evaluation und Rezertifizierung heute und in Zukunft;

- neue didaktische Methoden: E-Learning, Blended Learning, Simulation;

- diagnostic pédagogique pour les internes/assistants en difficulté de raisonnement clinique.

Im letzten Hauptreferat des Tages präsentierte Martin Fischer Ideen, wie «lebenslange ärztliche Kompetenzentwicklung in Zeiten ökonomischen Drucks» aussehen könnte. Der Vorsteher des Instituts für Didaktik und Ausbildungsforschung in der Medizin an der Universität München sieht die Zukunft in flexibleren Weiterbildungskonzepten und in einer kompetenzbasierten Ausund Weiterbildung. Diese erfordere allerdings ein dynamischeres Rollenverständnis von Lernenden und Lehrern. Den ökonomischen Druck bewertete er nicht nur negativ, da ökonomische Überlegungen als Argument für innovative Weiterbildungskonzepte dienen könnten.

«Haben Sie Ihrer Tochter empfohlen, Ärztin zu werden?», fragte Iwan Rickenbacher seine Gäste zum Auftakt des abschliessenden Podiumsgesprächs zum Thema «Die heutigen Herausforderungen und ihre Konsequenzen für die ärztliche Bildung». Dem Honorarprofessor für politische Kommunikation der Universität Bern gelang es, von den Diskussionsteilnehmern Carlo Conti, Markus Furrer, Jürg Hodler, Claudia Meuli-Simmen, Reto Meuli und Ryan Tandjung zu zentralen Themen des Tages pointierte Stellungnahmen zu erhalten und damit eine für das Publikum relevante und anregende Veranstaltung würdig abzuschliessen. 